\title{
New Direct Observational Evidence for Kicks in SNe
}

\author{
T. M. Tauris and E. P. J. van den Heuvel
}

CHEAF, and Astronomical Institute "Anton Pannekoek", University of Amsterdam, Kruislaan 403, 1098 SJ Amsterdam, The Netherlands

\section{Introduction}

We present an updated list of direct strong evidence in favour of kicks being imparted to newborn neutron stars. In particular we discuss the new cases of evidence resulting from recent observations of the X-ray binary Circinus X-1 and the newly discovered binary radio pulsar PSR J1141-6545. We conclude that the assumption that neutron stars receive a kick velocity at their formation is unavoidable (van den Heuvel \& van Paradijs 1997).

This assumption explains a large variety of observations, ranging from direct observed properties of individual binary pulsars and Be/X-ray binaries to the observed birth rates and dynamical properties of the populations of LMXBs, binary recycled pulsars as well as the motion and distribution of single pulsars. Below we give an updated list in favour of kicks based on the compilation given by van den Heuvel \& van Paradijs (1997) - see references therein for details.

\section{List of evidence for asymmetric supernovae and resulting kicks}

- High radial velocity of Circinus X-1 (Tauris et al. 1999), cf. Sect. 3

- Nonalignment of spin and orbit in PSR J0045-7319 (Kaspi et al. 1996)

- Geodetic precession and evolution of PSR 1913+16 (Wex et al. 2000)

- Low eccentricity of PSR J1141-6545 (Tauris \& Sennels 2000), cf. Sect. 4

- High eccentricities of Be/X-ray binaries (Verbunt \& van den Heuvel 1995)

- High velocities of certain BMSP/LMXBs (e.g. Tauris \& Bailes 1996)

- Population synthesis/incidence arguments (e.g. Dewey \& Cordes 1987)

The origin of the kick mechanism is still rather unclear; it either may be related to a neutrino driven convection instability and an asymmetric outflow of neutrinos or to mass outflow in a MHD jet during/following the core collapse.

\section{Circinus X-1 - survivor of a highly asymmetric SN}

Based on the recently measured (Johnston, Fender \& Wu 1999) radial velocity of $+430 \mathrm{~km} \mathrm{~s}^{-1}$, Tauris et al. (1999) find that a minimum neutron star kick velocity of $\sim 500 \mathrm{~km} \mathrm{~s}^{-1}$ is needed to account for such a high system radial velocity (they find that on average, a kick of $\sim 740 \mathrm{~km} \mathrm{~s}^{-1}$ is necessary). This is by far the largest kick needed to explain the motion of any observed binary system. It should be noted that this result is independent on the uncertainty of the exact mass of the companion star (most likely $1<M_{2} / M_{\odot}<2$ ). 


\section{PSR J1141-6545 - a young pulsar with an old deg. companion}

The very recently discovered non-recycled binary pulsar PSR J1141-6545 (Manchester et al. 1999) has a massive companion $\left(M_{2} \geq 1.0 M_{\odot}\right)$ in an eccentric 0.198 days orbit. The pulsar's high value of $\dot{P}_{\text {spin }}\left(4.31 \times 10^{-15}\right)$ in combination with its relatively slow rotation rate $\left(P_{\text {spin }}=394 \mathrm{~ms}\right)$ and non-circular orbit $(e=0.17)$ identifies this pulsar as being young and the last formed member of a double degenerate system. Given $P_{\text {orb }}=0.198$ days it is evident that a non-degenerate star can not fit into the orbit without filling its Roche-lobe. Based on evolutionary considerations and population synthesis, Tauris \& Sennels (2000) demonstrate that the companion is most likely to be an O-Ne-Mg white dwarf. In that case the system resembles PSR B2303+46: the first neutron star - white dwarf binary system observed, in which the neutron star was born after the formation of the white dwarf (van Kerkwijk \& Kulkarni 1999). The existence of a minimum eccentricity for systems undergoing a symmetric $\mathrm{SN}$ follows from celestial mechanics (e.g. Flannery \& van den Heuvel 1975): $e=\left(M_{2 \mathrm{He}}-M_{\mathrm{NS}}\right) /\left(M_{\mathrm{WD}}+M_{\mathrm{NS}}\right)$

If one assumes $M_{2 \mathrm{He}}>M_{\mathrm{He}}^{\text {crit }} \simeq 2.5 M_{\odot}, M_{\mathrm{WD}}^{\max }<1.4 M_{\odot}$ and $M_{\mathrm{NS}}=1.3 M_{\odot}$ it follows that $e>0.45$ (Note, that this result also remains valid if the companion star should turn out to be another neutron star with a similar mass). In order to reproduce the low observed eccentricity $(e=0.17)$ Tauris \& Sennels (2000) conclude that the neutron star must have received a natal kick velocity at birth of $>100 \mathrm{~km} \mathrm{~s}^{-1}$. The evidence of asymmetry remains even if one assumes an extremely low mass for the exploding naked helium star (e.g. adopting $M_{\mathrm{He}}^{\text {crit }}=2.0 M_{\odot}$ results in a minimum post-SN eccentricity of 0.26 ).

\section{References}

Dewey R. J., Cordes J. M., 1987, ApJ. 321, 780

Flannery B.P., van den Heuvel E.P.J., 1975, A\&A 39, 61

Johnston H. M., Fender R. P., Wu K., 1999, MNRAS 308, 415

Kaspi et al., 1996, Nature, 381, 584

Manchester R.N., et al., 1999, to appear in: IAU Colloq. 177, Pulsar Astronomy -2000 and Beyond, eds: M. Kramer et al. (ASP Conf. Series)

Tauris T.M., Bailes M., 1996, A\&A 315, 432

Tauris T.M., Sennels T., 2000, A\&A submitted (astro-ph/9909149)

Tauris et al., 1999, MNRAS 310, 1165

van den Heuvel E.P.J., van Paradijs J., 1997, ApJ. 483, 399

van Kerkwijk M. H., Kulkarni S. R., 1999, ApJ. 516, L25 ApJ. 204, L29

Verbunt F., van den Heuvel E. P. J., 1995 in: X-ray Binaries, eds: W.H.G. Lewin, J. van Paradijs and E.P.J. van den Heuvel (Cambridge), 457

Wex N., Kalogera V., Kramer M., 2000, ApJ, 528, 401 